\title{
Soluble human leukocyte antigen-G evaluation in pregnant women with gestational diabetes mellitus
}

\author{
Muhamad R. Abdel Hameed ${ }^{1 *}$ D, Osama Ahmed Ibrahiem', Entsar Hamed Ahmed², Paula Rofaeel Sedky ${ }^{3}$ and \\ Naglaa Mohamed M. A. Mousa ${ }^{3}$
}

\begin{abstract}
Background: Gestational diabetes mellitus is any degree of glucose intolerance with onset or first recognition occurring late in second trimester and third trimester of pregnancy. It constitutes a greater impact on diabetes epidemic as it carries a major risk for developing type 2 diabetes mellitus to the mother and her fetus later in life. human leukocyte antigen (HLA)-G is a class Ib gene presents in the human major histocompatibility complex (MHC). HLA-G has an important role for mother and fetus tolerance during pregnancy, also in the pancreatic islet cells protection. This is a case-control study, measuring serum HLA-G levels by ELISA in 60 pregnant women with gestational diabetes compared with 36 normal pregnant women.

Results: HLA-G levels were significantly high in pregnant women with gestational diabetes mellitus (GDM) in contrast to women with normal pregnancy $(P=0.001)$.

Conclusion: Women with GDM had significantly higher levels of soluble HLA-G than women without GDM, suggesting that HLA-G molecule is among the factors for regulation and control of the immune response and the induction of tolerance. Soluble HLA-G could be considered an important follow-up investigation for all pregnant primary health care for early detection of gestational diabetes.
\end{abstract}

Keywords: HLA-G, Gestational diabetes mellitus (GDM), Pregnancy

\section{Background}

Diabetes mellitus is a chronic illness that needs continuing self-management education and support for prevention of acute complications and reduction of chronic complications. Diabetes care requires multifactorial risk reduction strategies beyond glycemic control [1].

Gestational diabetes (GDM) has been defined as a degree of glucose intolerance with onset or first recognition during pregnancy including women whose glucose intolerance develop during pregnancy and preexisting diabetes which had not been diagnosed before pregnancy. The most unborn babies of women had glucose

\footnotetext{
* Correspondence: dr.muhamadramadan@yahooo.com

1 Department of Internal Medicine \& Clinical Hematology Unit, Faculty of Medicine, Assiut University, Assiut, Egypt

Full list of author information is available at the end of the article
}

intolerance in pregnancy; those of women with preexisting diabetes can be exposed to hyperglycemia in the first two trimesters of pregnancy resulting in an increased risk of a range of cardiovascular, central nervous system, and musculoskeletal defects. The consensus panel of the international association of diabetes and pregnancy study groups (IADPSG) recommended that high-risk women found to have diabetes at their initial prenatal visit which should be diagnosed as having overt diabetes rather than GDM [2].

GDM carries a major risk of developing T2DM to the mother and fetus later in life. GDM has been linked with the development of cardiovascular disease and metabolic syndrome. The cardinal cause of type 2 diabetes is genetic factors with lack of exercise, obesity caused by a high-fat diet, stress, and aging impairing insulin action, 
leading to the onset of diabetes. GDM incidence increases by approximately eight times for pregnant women aged 35 years and over compared with women aged 25 years or under. The GDM definition therefore does not include overt diabetes in pregnancy [3].

Recommended screening for GDM at 24-28 weeks of gestation by oral glucose tolerance tests (OGTTs). However, the current approach of diagnosis and treatment during the late second or early third trimester of pregnancy using OGTTs fails to prevent the "pathology" that is already well established and the adverse effects on long-term outcomes. Previous studies have identified early blood-borne biomarkers that may be associated with the development of GDM, including plasma glucose, placental growth factor (PGF), insulin, pregnancy associated plasma protein, and sex hormone binding globulin (SHBG) [4].

Usually, symptoms of diabetes disappear after delivery. The cause of GDM is still unknown, and hormonal status in pregnancy, particularly human placental lactogen, is associated with gestational diabetes, but the exact reason why gestational diabetes occurs in some pregnant women is unknown [5].

Human leukocyte antigen (HLA)-G is a class Ib gene located in the human major histocompatibility complex (MHC) that is located at 16p21.3 location on chromosome 6 [6].

HLA-G molecule was first detected on placental cytotrophoblasts and involved in maternal acceptance of the semi-allogenic fetus in pregnancy and in the development of tolerance. Soluble HLA-G (sHLA-G) is positively correlated with successful treatment of in vitro fertilization (IVF). Aberrant expression of HLA-G was observed in some gestational complications, like spontaneous abortion and pre-eclampsia [7].

\section{Aim of the work}

Evaluation of the association of soluble HLA-G in pregnant women with gestational diabetes compared with healthy pregnant women, emphasizing the necessity of using such parameter as a routine follow-up for pregnant women for early detection of GDM so, avoiding diabetic compilations.

\section{Methods}

The current study was performed at the Gestational Diabetes Mellitus (GDM) Outpatient Clinic of Assuit University hospitals in period between November 2018 and November 2019. The study enrolled 96 pregnant women in 24th to 28th age of gestation. Through clinical examination and history taking were performed with exclusion of those having past history of diabetogenic drug medication or endocrine diseases causing secondary diabetes. Exclusion criteria include chronic hepatitis $\mathrm{C}$ and liver cirrhosis. Enrolled women were subdivided into two groups based on oral glucose tolerance test and World Health Organization recommendation for GDM diagnosis by exceedance of one or more of the following three plasma glucoses: fasting plasma glucose $\geq 5.1$ $\mathrm{mmol} / \mathrm{L}(\geq 92 \mathrm{mg} / \mathrm{dL}), 1 \mathrm{~h} \geq 10.0 \mathrm{mmol} / \mathrm{L}(180 \mathrm{mg} / \mathrm{dL})$, or $2 \mathrm{~h} \geq 8.5 \mathrm{mmol} / \mathrm{L}(153 \mathrm{mg} / \mathrm{dL})[8]$ :

- Study group included 60 pregnant women with GDM.

- Control group included 36 pregnant women without GDM

\section{ELISA (enzyme-linked immunosorbent assay):}

Two milliliters of blood was collected after centrifugation serum was extracted. We measured serum soluble HLA-G by commercial Human MHCG/HLA-G (Major Histocompatibility Complex Class I - G) ELISA Kit (Elabscience ${ }^{\circ}$ ) according to kit protocol (Sandwich-ELISA principle).

\section{Reagent preparation}

1- All reagents were brought to room temperature $\left(18-25^{\circ} \mathrm{C}\right)$. The microplate was preheated for 15 min before OD measurement.

2- Wash buffer: $30 \mathrm{ml}$ of concentrated wash buffer was diluted with $720 \mathrm{ml}$ of deionized or distilled water to prepare $750 \mathrm{~m}$ of wash buffer.

Standard working solution: the standard was centrifuged at $10,000 \times g$ for $1 \mathrm{~min}$. One milliliter of reference standard and sample diluent was added and stood for 10 min and inverted gently several times. After it dissolved fully, it was mixed thoroughly with a pipette.

This reconstitution produces a working solution of 20 $\mathrm{ng} / \mathrm{ml}$. This made serial dilutions as follows: $20 \mathrm{ng} / \mathrm{ml}$, $10 \mathrm{ng} / \mathrm{mL}, 5 \mathrm{ng} / \mathrm{mL}, 2.5 \mathrm{ng} / \mathrm{mL}, 1.25 \mathrm{ng} / \mathrm{mL}, 0.63 \mathrm{ng} /$ $\mathrm{mL}, 0.31 \mathrm{ng} / \mathrm{mL}$, and $0 \mathrm{ng} / \mathrm{mL}$.

\section{Assay procedure}

1- The standard working solution was added to the first two columns. Each concentration of the solution is added in duplicate, to one well each, side by side (100 uL for each well). The samples were added to the other wells (100 uL for each well). The plate was covered with sealer provided in the kit, then incubated for $90 \mathrm{~min}$ at $37^{\circ} \mathrm{C}$.

2- The liquid was removed out of each well, did not wash, and immediately $100 \mu \mathrm{L}$ of Biotinylated Detection Ab working solution was added to each well, then covered with plate sealer and then mixed up and incubated for $1 \mathrm{~h}$ at $37^{\circ} \mathrm{C}$. 
3- The solution was aspirated from each well, $350 \mu \mathrm{l}$ of wash buffer was added to each well and soaked for 1-2 min, and the solution was aspirated from each well and was put it dry against clean absorbent paper. Wash step was repeated 3 times in total.

4- One hundred microliters of HRP conjugate working solution was added to each well, then covered with plate sealer and incubated for $30 \mathrm{~min}$ at $37^{\circ} \mathrm{C}$.

5- The solution was aspirated from each well. The wash process was repeated for five times as conducted in step 3.

6- Ninety microliters of substrate reagent was added to each well, then covered with a new plate sealer and incubated for $15 \mathrm{~min}$ at $37^{\circ} \mathrm{C}$.

7- Fifty microliters of stop solution was added to each well.

8- The optical density (OD value) of each well was determined at once, using micro-plate reader set at $450 \mathrm{~nm}$.

\section{Statistical analysis}

Collected data was analyzed with those using SPSS (Statistical Package for the Social Science, version 20, IBM, and Armonk, New York). Continuous data was expressed in form of mean \pm SD or median (range) while nominal data was expressed in form of frequency (percentage).

$\mathrm{Chi}^{2}$-test was used to compare the nominal data of different groups in the study while student's $t$ test was used to compare mean of different two groups. The receiver operating characteristic (ROC) curve was used to determine the diagnostic accuracy of HLA-G in diagnosing gestational diabetes mellitus. Level of confidence was kept at 95\%, and hence $P$ value was significant if $P<$ 0.05 .

\section{Results}

Baseline data of enrolled women is shown in Table 1 . Mean age of women with GDM was $31.13 \pm 6.41$ years and with a mean gestational age which was $25.90 \pm 1.52$ weeks while mean age of women without GDM was $30.51 \pm 6.97$ years with mean gestational age which was $26.14 \pm 1.53$ weeks with no significant differences between groups as regarding age and gestational age ( $P$ $=0.66$ and 0.45 , respectively).

It was noticed that mean body mass index was significantly higher among those women with GDM in comparison to the control group $(26.56 \pm 5.55$ vs. $21.12 \pm$ $\left.4.56 \mathrm{~kg} / \mathrm{m}^{2} ; P=0.01\right)$. Also, family history of diabetes mellitus (DM) was significantly higher among those with GDM (8 (13.33\%) vs. 2 (5.5\%); $P=0.04)$.

Both groups had insignificant differences as regarding age, gestational age, parity, and frequency of abortion $(P$ $>0.05$ ).

\section{Level of sHLA-G in enrolled women}

The current study showed that pregnant women with GDM had significantly higher HLA-G in comparison to those without GDM $(1.93 \pm 0.19 \mathrm{ng} / \mathrm{mL}$ vs. $0.21 \pm 0.16$ ng $/ \mathrm{mL} ; P<0.001$ ) (Fig. 1).

\section{Diagnostic accuracy of HLA-G in diagnosing GDM}

It was noticed that for diagnosis of GDM, HLA-G at cutoff point $>0.7$ had $94 \%$ sensitivity and $91 \%$ specificity with overall accuracy of 93\% (Table 2) (Fig. 2).

\section{Discussion}

Maternal age is an established risk factor for gestational diabetes mellitus (GDM), but there is no consensus on the age above which there is significantly increased risk of GDM [9]. In the present study, the mean age of the women with GDM was $31.13 \pm 6.41$ years.

In concordance with our findings, Khan and colleagues [10] reported GDM socio demographic risk factors in Khyber Teaching Hospital in Pakistan. A total of 103 GDM and 97 healthy pregnant women were included. The mean age of the included women was $31.29 \pm 5.79$ years old.

Similarly, Egbe and colleagues [11] conducted a crosssectional study which was carried out in Limbe, Cameroon. Women with GDM had a mean age of 27.8 (SD 5.7) years.

Likewise, Khanna and colleagues [12] aimed to document the longitudinal characteristics of GDM women for demographic insights. GDM pregnancy charts were assessed for 2000-2002, 2010-2012, and

Table 1 Baseline data of enrolled women

\begin{tabular}{llll}
\hline & Study group $(\boldsymbol{n}=60)$ & Control group $(\boldsymbol{n}=36)$ & $\boldsymbol{P}$ value \\
\hline Age (year) & $31.13 \pm 6.41$ & $30.51 \pm 6.97$ & 0.66 \\
Gestational age (week) & $25.90 \pm 1.52$ & $26.14 \pm 1.53$ & 0.45 \\
Parity & $2(1-5)$ & $2(1-4)$ & 0.51 \\
Abortion & $1(1-2)$ & $1(0-1)$ & 0.65 \\
Body mass index (k/m2) & $26.56 \pm 5.55$ & $21.12 \pm 4.56$ & 0.01 \\
Family history of DM & $8(13.33 \%)$ & $2(5.5 \%)$ & 0.04 \\
\hline
\end{tabular}

Data expressed as frequency (percentage), mean (SD), and median (range). $P$ value was significant if $<0.05$. DM diabetes mellitus 


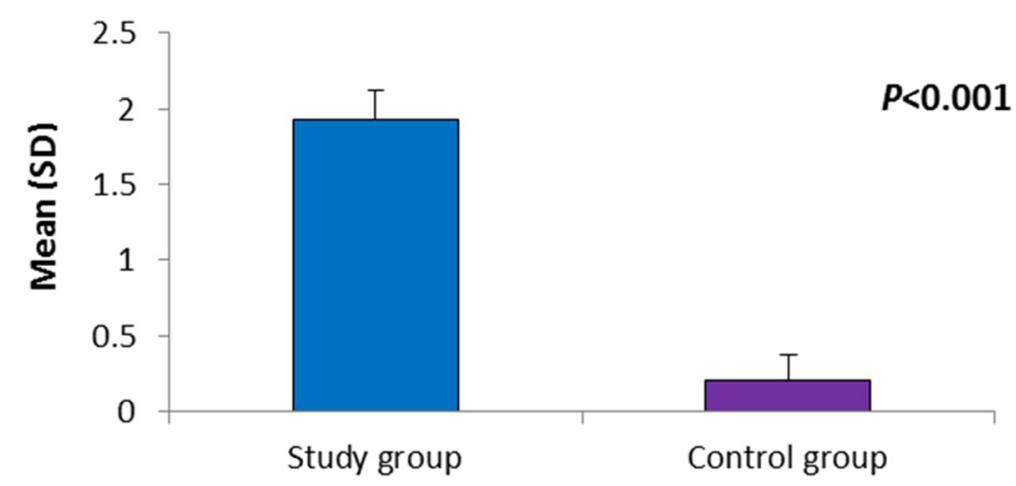

Fig. 1 Level of sHLA-G in enrolled women $(n g / m L)$

2014-2016. The mean age of included women was $33.1 \pm 5.6$ years old.

Obesity is an important risk factor for GDM. The rate of GDM appears to be 2-fold higher in obese women than non-obese women [13].

In the present study, the mean body mass index was significantly higher among those women with GDM in comparison to the control group (26.56 \pm 5.55 vs. 21.12 $\pm 4.56 \mathrm{~kg} / \mathrm{m} 2 ; P=0.01$ ).

In agreement with our findings, Martin and colleagues [14] evaluated the effect of maternal BMI on GDM and the risk of gestational complications in obese or overweight women. A total of 1030 women were included between 10 and 20 weeks of gestation with a BMI $\geq 25$ $\mathrm{kg} / \mathrm{m}(2)$ and were grouped into BMI subclasses utilizing the WHO criteria. The prevalence of GDM increased with increased maternal BMI $(6.74 \%$ overweight vs $13.42 \%$ obese subclass 1 vs $12.79 \%$ obese subclass 2 vs 20.00\% obese subclass 3).

Similarly, Najafi and colleagues [15] investigated the effect of pre-pregnancy BMI on the risk of GDM. Five electronic databases, including PubMed, Scopus, Embase, Web of Science, and Google Scholar, had searched for literatures published until 1 January 2018. A total of 33 observational studies, with sample size of 962,966 women and 42,211 patients with GDM, were

Table 2 Diagnostic accuracy of HLA-G in diagnosing GDM

\begin{tabular}{ll}
\hline Parameters & Value \\
\hline Sensitivity & $94 \%$ \\
Specificity & $91 \%$ \\
Positive predictive value & $95 \%$ \\
Negative predictive value & $88 \%$ \\
Accuracy & $93 \%$ \\
Cutoff point & $>0.70$ \\
$P$ value & $<0.001$ \\
\hline Pvale
\end{tabular}

$P$ value was significant if $<0.05$. GDM gestational diabetes mellitus included. The increase of GDM risk was $4 \%$ per unit of BMI increase.

Additionally, Emerson and colleagues [16] examined the utility of BMI as a screening tool for GDM across ethic subgroups. All results showed that rates of GDM increased consistently with increasing BMI.

Diabetes family history is considered as a risk factor for gestational glucose intolerance. Family history is an indication of oral glucose tolerance test [17].

In our cohort, family history of DM was significantly higher among those with GDM than control groups (8 (13.33\%) vs. 2 (5.5\%); $P=0.04)$.

In concordance with our findings, Moosazadeh and colleagues [18] conducted a meta-analysis to determine the relationship between the GDM and family history of diabetes. A total of 33 relevant studies were included in meta-analysis process including 2697 women with family history of diabetes mellitus in addition to 29134 women without. The overall odds ratio of family history for developing GDM was estimated as of 3.46 (95\% CI 2.80-4.27).

Likewise, El Sagheer and Hamdi [19] assessed the prevalence of GDM in El-Minya Governorate, Egypt, using the Diabetes in Pregnancy Study Group India (DIPSI) in comparison to the International Association of Diabetes and Pregnancy Study Groups (IADPSG) criteria, and assessment of the risk factors of GDM in such locality. There were 700 pregnant women underwent the 75 -g oral glucose tolerance test irrespective of the meal and plasma glucose measurement after $2 \mathrm{~h}$. The multiple logistic regression analysis has shown that family history of DM and BMI was determined as independent risk factors of GDM.

Regarding the primary outcome of the present study, the current study showed that pregnant women with GDM had significantly higher HLA-G in comparison to those without GDM $(1.93 \pm 0.19$ vs. $0.21 \pm 0.16$; $P<0.001)$. It was noticed that for diagnosis of GDM, HLA-G at cutoff point > 0.7 had $94 \%$ sensitivity and $91 \%$ specificity with overall accuracy of $93 \%$. 


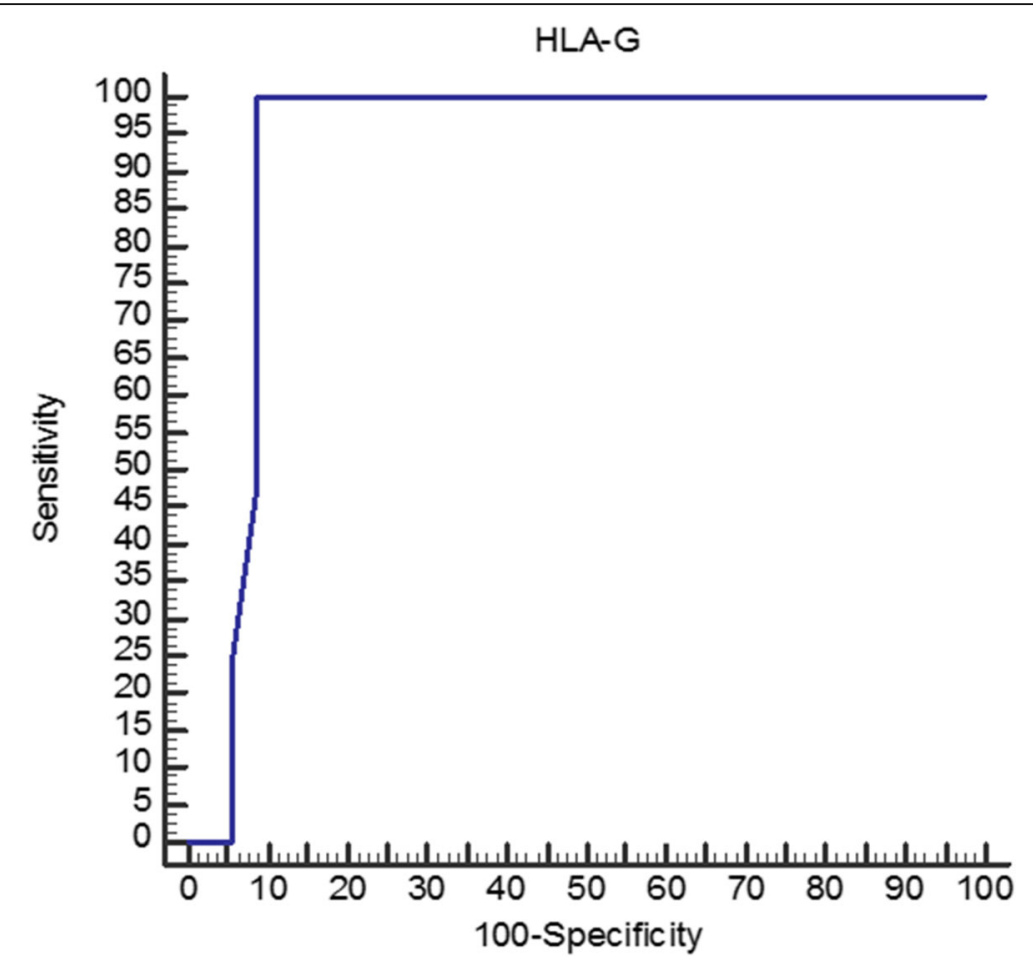

Fig. 2 Diagnostic accuracy of HLA-G in diagnosing GDM

In concordance with our findings, Martinetti and colleagues [20] evaluated 151 non-GDM women and 26 pregnant women with GDM. HLA-G plasma levels were measured by ELISA method at first and second trimesters of pregnancy, at delivery, and in cord blood. GDM mothers showed increased levels of sHLA-G compared to controls $(p=0.004)$, and those carrying the HLA-G del/ del genotype produced more sHLA-G at the second trimester and at delivery $(p=0.014)$.

Similarly, Veiga-Castelli and colleagues [21] evaluated 102 GDM and 150 non-GDM women to detect HLA-C, $-\mathrm{E}$, and $-\mathrm{G}$ polymorphisms and haplotypes. They observed 14 allelic groups when the HLA-C gene was evaluated, and two of them (HLA-C/05, mainly represented by the C/05: 01:01, and HLA-C/08, mainly represented by the allele C/ 08:02:01) were associated with protection against GDM.

Association between HLA-G and GDM is not fully understood. However, this could be related to the activity of these molecules in regulating the immune system. Normal concentrations of these molecules in normal pregnant women may be indicative of immune response inhibition, and the regulatory role of this molecule in pregnancy and higher values in pregnant women with gestational diabetes may be an indicative of removal of this inhibition and regulation. It is suggested that this assumption can be the reason for developing GDM in these women comparing to the normal control group [22].
In contrary to our findings, Shobeiri and colleagues [23] investigated the concentration of HLA-G in pregnant women with GDM in comparison with normal pregnant women. In such case-control study, the authors measured serum HLA-G levels in 24 pregnant women with GDM compared with 30 normal pregnant women using sandwich ELISA technique. HLA-G levels were significantly low in pregnant women with gestational diabetes in contrast to normal pregnant women $(P=0.001)$.

The exact causes of such heterogeneity between our findings and those studies which reported lower levels of HLA-G in GDM are unclear. However, these differences can be attributed to the difference in population's characteristics, different in measurements' methods, or different study designs.

\section{Study's limitations}

We acknowledge that the present study results cannot be generalized to the general Egyptian population. The descriptive nature of the present study may also preclude the conclusion of association analysis.

\section{Recommendation}

HLA-G could be tested in pregnant women and may be as an effective molecule to take an important step in the prediction of gestational diabetes to prevent problems and consequences of this disease. 


\section{Conclusion}

Women with GDM had significantly higher levels of soluble HLA-G than women without GDM, suggesting that HLA-G molecule is among the factors for regulation and control of the immune response and the induction of tolerance. Soluble HLA-G must be used as an important follow-up investigation for all pregnant primary health care for early detection of gestational diabetes. Further, studies with rigorous design, large sample size, and multiregional cooperation are required.

\author{
Abbreviations \\ DM: Diabetes mellitus; GDM: Gestational diabetes mellitus; HLA-G: Human \\ leucocyte antigen-G
}

\section{Acknowledgements}

"Not Applicable" in this section.

\section{Authors' contributions}

M.R.A.H., N.M.M.A.M., O.A.I., E.H.A., and P.R.S. conceived and designed the research. M.R.A.H, P.R.S., and N.M.M.A.M. recruited patients, carried out the clinical investigations, and collected patients' clinical data. E.H.A. performed the experiments. M.R.A.H., N.M.M.A.M, O.A.I., E.H.A., and P.R.S. prepared the original draft of the manuscript; participated in data analysis and writing, and read and agreed to the published version of the manuscript. The authors read and approved the final manuscript.

\section{Funding}

The research was funded by authors

\section{Availability of data and materials}

The data that support the findings of this study are available from the corresponding author upon reasonable request.

\section{Ethics approval and consent to participate}

This study was approved by the Regional Ethics Committee, Faculty of Medicine, Assuit University and was conducted in accordance with the provisions of the Declaration of Helsinki. Approval number 17101085 and Date: November 16, 2016.

\section{Consent for publication}

Not applicable

\section{Competing interests}

The authors declare that there are no conflicts of interest.

\section{Author details}

${ }^{1}$ Department of Internal Medicine \& Clinical Hematology Unit, Faculty of Medicine, Assiut University, Assiut, Egypt. ${ }^{2}$ Department of Medical Microbiology \& Immunology, Faculty of Medicine, Assiut University, Assiut, Egypt. ${ }^{3}$ Department of Internal Medicine, Faculty of Medicine, Assiut University, Assiut, Egypt.

Received: 13 May 2020 Accepted: 3 July 2020

Published online: 03 November 2020

\section{References}

1. Sanders of medical care in diabetes. American diabetes association, care diabetes journal org; 2013.

2. Clive J, Petry. Gestational diabetes: risk factors and recent advances in genetics and treatment. Br J Nutr 2010;104:775-787.

3. Kim C, Newton KM, Knopp RH (2002) Gestational diabetes and incidence of type 2 diabetes: a systematic review. Diabetes Care 25:1862-1868

4. Meiro Y, Albers D, Rice G, Roa J, Cortez J,Monckeberg M, Schepeler M, Osorio E, Illanes SE (2019) First trimester prediction of gestational diabetes mellitus using plasma biomarkers: a case-control study. J Perinat Med 47(2): $161-168$
5. Öztekin Ö (2007) New insights into the pathophysiology of gestational diabetes mellitus: possible role of human leukocyte antigen-G. Med Hypotheses 69:526-530

6. Abediankenari S, Ghasemi M, Kim YJ (2011) Human leukocyte antigen-G expression on dendritic cells induced by transforming growth factor-beta1 and CD4+ T cells proliferation. Iran Biomed J 15:1-5

7. Abediankenari S, Ghasemi M (2009) Generation of immune inhibitory dendritic cells and CD4 + T regulatory cells inducing by TGF-beta. Iran J Allergy Asthma Immunol 8:25-30

8. World Health Organization (2014) Diagnostic criteria and classification of hyperglycaemia first detected in pregnancy: a World Health Organization guideline. Diabetes Res Clin Pract 103:341-363

9. Kuo $\mathrm{CH}$, Chen SC, Fang CT, Nien FJ, Wu ET, Lin SY, Chuang LM, Lee CN, Li HY (2017) Screening of gestational diabetes mellitus: the role of maternal age. PLoS One 12(3):e0173049

10. Khan R, Ali K, Khan Z (2013) Sociodemographic risk factors of gestational diabetes mellitus. Pakistan J Med Sci 29(3):843

11. Egbe TO, Tsaku ES, Tchounzou R, Ngowe MN (2018) Prevalence and risk factors of gestational diabetes mellitus in a population of pregnant women attending three health facilities in Limbe, Cameroon: a cross-sectional study. The Pan African Medical Journal 31

12. Khanna P, Anukam K, Chow L, Brydges E, Liu SL, Mahon J, Joy TR, Mcmanus RM. 2412-PUB: Demographics of women with gestational diabetes attending a diabetes and pregnancy clinic: 2000-2002, 2010-2012, and 2014-2016.

13. Karim J, Ogunnoiki W, Perera A (2010) BMI threshold for gestational diabetes: a survey assessing sensitivity of BMl in screening for women with gestational diabetes mellitus. Arch Dis Child Fetal Neonatal Ed 95(1):42-43

14. Martin KE, Grivell RM, Yelland LN, Dodd JM (2015) The influence of maternal BMI and gestational diabetes on pregnancy outcome. Diab Res Clin Practice 108(3):508-513

15. Najafi F, Hasani J, Izadi N, Hashemi-Nazari SS, Namvar Z, Mohammadi S, Sadeghi M (2019) The effect of pregnancy body mass index on the risk of gestational diabetes mellitus: a systematic review and dose-response metaanalysis. Obesity Rev 20(3):472-486

16. Emerson J, Main E, Gilbert W, Chung J, Melsop K, Cheng Y, Snowden J, Caughey A (2013) 238: BMl and gestational diabetes. Am J Obstetr Gynecol 208(1):S110

17. Chan LS, Wong S, Ho L (2002) Diabetic family history is an isolated risk factor for gestational diabetes after 30 years of age. Acta Obstet Gynecol Scand 81(2):115-117

18. Moosazadeh M, Asemi Z, Lankarani KB, Tabrizi R, Maharlouei N, Naghibzadeh-Tahami A, Yousefzadeh G, Sadeghi R, Khatibi SR, Afshari M, Khodadost M (2017) Family history of diabetes and the risk of gestational diabetes mellitus in Iran: a systematic review and meta-analysis. Diab Metab Syndr 11:S99-S104

19. El Sagheer GM, Hamdi L (2018) Prevalence and risk factors for gestational diabetes mellitus according to the diabetes in pregnancy study group India in comparison to international association of the diabetes and pregnancy study groups in El-Minya, Egypt. Egypt J Internal Med 30(3):131

20. Martinetti M, Beneventi F, Capittini C, Locatelli E, Simonetta M, Cavagnoli C, De Maggio I, De Silvestri A, Pasi A, Spinillo A (2017) The immunosignature of mother/fetus couples in gestational diabetes mellitus: role of hla-g 14 bp ins/del and papp-a a/c polymorphisms in the uterine inflammatory milieu. Dis Markers 2017

21. Veiga-Castelli LC, Collares CV, Porto IO, Moises E, Foss-Freitas MC, MendesJunior CT, Castelli EC, Donadi EA (2017) P014 HLA-C, HLA-E and HLA-G regulatory and coding region polymorphisms in patients exhibiting gestational diabetes mellitus. Hum Immunol 78:64

22. Shobeiri SS, Abediankenari S, Lashtoo-Aghaee B, Rahmani Z, Esmaeili-Gorji B (2016) Evaluation of soluble human leukocyte antigen-G in peripheral blood of pregnant women with gestational diabetes mellitus. Caspian J Internal Med. 7(3):178

23. Shobeiri SS, Abediankenari S, Lashtoo-Aghaee B, Rahmani Z, Esmaeili-Gorji B (2016) Evaluation of soluble human leukocyte antigen-G in peripheral blood of pregnant women with gestational diabetes mellitus. Caspian J Internal Med 7(3):178

\section{Publisher's Note}

Springer Nature remains neutral with regard to jurisdictional claims in published maps and institutional affiliations. 